\title{
Incidence of hypertension and risk of cardiovascular events among ethnic Chinese: report from a community-based cohort study in Taiwan
}

\author{
Kuo-Liong Chien ${ }^{a, b}$, Hsiu-Ching Hsu ${ }^{b}$, Fung-Chang Sung ${ }^{c}$, Ta-Chen Su $^{b}$, \\ Ming-Fong Chen ${ }^{b}$ and Yuan-Teh Lee ${ }^{b, d}$
}

Background Hypertension confers risk on cardiovascular events; however, less is known concerning hypertension incidence among ethnic Chinese. The effect of prehypertension on new onset of hypertension and cardiovascular events is also unknown.

Objectives To investigate the incidence of hypertension, risk factors and cardiovascular events in Taiwan.

Design A prospective community-based cohort design.

Setting and participants The Chin-Shan Community Cardiovascular Cohort consisted of $\mathbf{1 7 0 3}$ men and 1899 women aged 35 years old and above, of homogeneous Chinese ethnicity, from 1990 to 2005.

Main outcome measures Hypertension incidence rates, cardiovascular events.

Results The patterns of adjusted cumulative incidence probability were curvilinear, increasing from $1 \%$ in the first period to $27 \%$ in the fourth period in normotensive subjects. The patterns among prehypertensives were from $4 \%$ in the first period to $59 \%$ in the fourth period. Women had an $18 \%$ lower risk of developing hypertension than men. The

\section{Introduction}

Hypertension places a great burden on public health in the middle-aged to older population, and its high prevalence and low control rates make it a target for public health promotion. Much less is known about the incidence of newly developed hypertension than its prevalence. In the USA, the Framingham cohort provided information about risk factors for the incidence of hypertension [1]. In another nationwide survey, the National Health Epidemiologic Follow-Up study, agespecific incidence rates increased in patients of younger age, reaching a peak at 55-64 year of age, then decreasing in the older population [2]. In addition, incidence rates differ in different ethnic populations; those of African Americans are at least twice the values of Caucasian populations [2].

Although reports have provided data on hypertension incidence [3-8], several issues remain to be clarified. survival curves were distinctly different for different blood pressure categories $(P<0.001)$. Baseline blood pressure categories played important roles in predicting cardiovascular risks; the hazard risks of prehypertension and hypertension increased from 1.73 to 4.52 , compared with baseline normotensive subjects.

Conclusion The hypertension incidence rates among ethnic adult Chinese were high and were associated with obesity and metabolic syndrome status. Furthermore, the baseline prehypertension elicited significant risk of cardiovascular events in the community. $J$ Hypertens 25:1355-1361 @ 2007 Lippincott Williams \& Wilkins.

Journal of Hypertension 2007, 25:1355-1361

Keywords: Chinese, hypertension, incidence, prehypertension, risk factors

anstitute of Preventive Medicine, College of Public Health, National Taiwan University, ${ }^{b}$ Department of Internal Medicine, National Taiwan University Hospital, Taipei, 'Institute of Environmental Health, College of Public Health, China Medical University, Taichung and ${ }^{\mathrm{d}}$ Ming-Sheng Healthcare, Taoyuang, Taiwan

Correspondence to Yuan-Teh Lee MD, PhD, Department of Internal Medicine, National Taiwan University Hospital, Taipei, Taiwan, 100

Tel: +886 2 23562830; fax: +8862 2392 0456; e-mail: ytlee@ha.mc.ntu.edu.tw

Received 7 October 2006 Revised 23 January 2007

Accepted 30 January 2007

First, most study populations have been based on ethnic Caucasians and African Americans, and there are scant data on the ethnic Chinese population. Second, the subject number has been limited or the follow-up time has been relatively short [4,7]. Finally, the hypertension criteria in previous studies are obsolete, and the effects of prehypertension on new-onset hypertension, defined by the Seventh Joint National Committee [9], are still unknown.

This study, a community-based cohort study on ethnic Chinese, provides a unique opportunity to investigate the incidence of new-onset hypertension. The study subjects were of homogeneous ethnicity and lifestyles, and longitudinal follow-up data made causal inference possible. We investigated whether ethnic adult Chinese had high hypertension incidence rates and cardiovascular events in the community. We focused particularly on baseline prehypertension and related risk factors for predicting 
further hypertension incidence. We also investigated the roles of baseline blood pressure for the prognosis of cardiovascular events.

\section{Methods}

\section{Study design and study population}

The study design and entry criteria for the Chin-Shan Community Cardiovascular Cohort (CCCC) study have been described elsewhere [10-12]. In brief, the initial cohort consisted of 1703 men and 1899 women aged 35 years and above, of homogeneous Chinese ethnicity, from 1990 to 2005. In this study, we included 3575 subjects with available blood pressure data. The cardiologists from National Taiwan University Hospital conducted the medical examinations with the approval of the hospital institutional review committee. Inclusion criteria were age older than 35 years according to the available vital statistics data. In the baseline survey, all study participants were individually interviewed with a structured questionnaire. Trained medical students canvassed door-to-door with the assistance of community leaders to extend invitations for the baseline survey to collect information including socio-demographic characteristics, lifestyle behavior, dietary characteristics, and personal and family histories of diseases and hospitalizations. With the consent of participants, physicians and students conducted physical examinations and laboratory tests for those participants invited to the clinic.

\section{Baseline measurements}

Blood pressure was measured in the right arm twice, using a mercury sphygmomanometer, with the subject seated comfortably and arms supported and positioned at the level of the heart. A 12-lead electrocardiogram was also performed for each participant, and two cardiologists evaluated the results blindly. Body mass index (BMI) was calculated as weight (in $\mathrm{kg}$ )/height ${ }^{2}$ (in $\mathrm{m}^{2}$ ) [13]. The average of blood pressure measurements was used, as described previously $[11,14]$. All venous blood samples drawn after a 12-h overnight fast were immediately refrigerated and transported within $6 \mathrm{~h}$ to the National Taiwan University Hospital. Standard enzymatic tests for serum cholesterol and triglyceride were used (Merck, Darmstadt, Germany). High-density lipoprotein cholesterol (HDL-C) levels were measured in supernatants after the precipitation of specimens with magnesium chloride phosphotungstate reagents (Merck). Low-density lipoprotein cholesterol (LDL-C) concentrations were calculated as 'total cholesterol minus cholesterol in the supernatant' by the precipitation method (Merck) [12].

\section{Follow-up measurement}

We collected questionnaires and blood samples from the participants at baseline (1990-1991), the first follow-up period (1992-1993) and the fourth follow-up period
(1997-1998) [10]. We measured blood pressure and collected antihypertensive medication in the serial follow-up visit periods. Excluding subjects in the hypertension category during the baseline period (1990-1991), we calculated the cumulative incidence rates of hypertension biennially in the first three periods (1992-1993, 1994-1995, 1996-1997) and the 2000-2001 period. The response rates in all periods were relatively high, from 86 to $96 \%$.

\section{Classification of blood pressure categories and associated risk factors}

We classified the blood pressure categories according to the criteria established by the Seventh Joint National Committee [9]. Normotensive was defined as systolic blood pressure (SBP) less than $120 \mathrm{mmHg}$ and diastolic blood pressure (DBP) less than $80 \mathrm{mmHg}$. Prehypertension was defined as SBP between 120 and $139 \mathrm{mmHg}$ or DBP between 80 and $89 \mathrm{mmHg}$. Stage 1 hypertension was defined as SBP between 140 and $159 \mathrm{mmHg}$ or DBP between 90 and $99 \mathrm{mmHg}$, and stage 2 hypertension was defined as SBP $\geq 160 \mathrm{mmHg}$ or DBP $\geq 100 \mathrm{mmHg}$ [9]. Subjects with hypertensive therapy were included. Individuals with a fasting blood sugar level higher than $126 \mathrm{mg} / \mathrm{dl}$ and/or using oral hypoglycemic agents or insulin injections were defined as having diabetes mellitus. Metabolic syndrome was defined by the Adult Treatment Panel III guidelines [15], with the waist circumference modified by Asian criteria [16]. For original Adult Treatment Panel III criteria, a participant with three of following was defined as having metabolic syndrome: blood pressure of at least $130 / 85 \mathrm{mmHg}$; serum triglyceride of at least $150 \mathrm{mg} / \mathrm{dl}$; HDL-C $<40 \mathrm{mg} / \mathrm{dl}$ in men and $<50 \mathrm{mg} / \mathrm{dl}$ in women; fasting glucose of $110 \mathrm{mg} / \mathrm{dl}$ or more; and central obesity, waist circumference greater than $102 \mathrm{~cm}$ in men and $88 \mathrm{~cm}$ in women. Modified Asian criteria are the same as original Adult Treatment Panel III criteria with the exception that waist circumference is greater than $90 \mathrm{~cm}$ in men and $80 \mathrm{~cm}$ in women [16]. Smoking habit was defined by current smoking status. We defined drinking status as a binary variable by frequency of drinking habits. Left ventricular hypertrophy was defined by voltage criteria via electrocardiogram. Hypercholesterolemia was defined as total cholesterol of at least $240 \mathrm{mg} / \mathrm{dl}$.

\section{Endpoint follow-up and disease verification}

The study team conducted biennial follow-up household visits with the study participants, and death certificates were reviewed monthly at the local vital statistics office. The adjudication procedure for cardiovascular events, including coronary heart disease, stroke and death, is reported elsewhere $[11,14]$. In brief, the definition of coronary events includes coronary death, nonfatal myocardial infarction, and hospitalization due to procedures of coronary artery bypass graft and percutaneous coronary intervention. A stroke event was defined as death from 
stroke or hospitalization due to sudden neurological deficit of vascular origin. The procedures were reviewed and adjudicated by a panel of trained physicians.

\section{Statistical analysis}

Continuous variables are presented as the mean and standard deviation or standard error of the mean, and binary variables are presented as contingency tables. We stratified the analysis based on gender and baseline blood pressure categories. We analyzed two endpoints in this study.

First, we investigated the new onset of hypertension in serial visit periods. We included the numbers of the participants who were not hypertensive in baseline measurement (1237 for men and 1271 for women), after excluding hypertensive subjects $(n=1070)$. We calculated the incidence rates in the serial follow-up visit periods. Because the information on whether or not hypertension onset was detected at any time up to the last visit was recorded, the incidence of hypertension was considered as interval-censored data. Interval censoring is a situation where the event happens between two visit periods [17]. We modeled hypertension incidence probability in the follow-up periods by the complementary $\log -\log$ binary regression model [18]. We estimated the visit period effects and covariates to predict the hypertension onset, calculating the cumulative hypertension incidence rates using the fitted model, specified by baseline normotensive and prehypertensive status. The odds ratios and 95\% confidence intervals of various risk factors for hypertension incidence probability were estimated with the complementary $\log -\log$ regression model.

Second, we estimated cardiovascular event rates at specific blood pressure levels. The person-year method was used to calculate the incidence rates of cardiovascular disease events, and survival curves were estimated according to baseline blood pressure categories, using the KaplanMeier procedure. The curves were compared with the log-rank test among these categories [19]. The multivariate Cox proportional hazard model was used to provide point and interval estimates of the hazard risk of cardiovascular events with blood pressure categories and various risk factors [20]. The adjusted variables included age, gender, BMI, diabetes mellitus, hypercholesterolemia, left ventricular hypertrophy, smoking and alcohol drinking habits in the model. Dummy variables were used to estimate the hazard risks of various blood pressure categories, compared with baseline normal blood pressure. We adopted a robust inference of covariance structure estimation in the model due to the fact that a robust covariance structure was appropriate or practical in use with a small number of events [21]. In addition, the proportional-hazards assumptions were checked by plotting, and the statistics based on Schoenfeld residuals were tested [22]. All data analyses were performed using SAS (version 9.1; SAS Institute Inc., Cary, North Carolina, USA) and STATA (version 9.1; StataCorp LP., College Station, Texas, USA) statistical software.

\section{Results}

The gender-specific mean levels and prevalence of risk factors according to gender and the baseline blood pressure categories of the 3575 subjects recruited in the cohort are presented in Table 1. Among men, the mean values of BMI, and total and LDL-C increased Table 1 Age-adjusted mean levels and prevalence of socio-
economic status, lifestyle patterns and various risk factors according to gender and baseline blood pressure

\begin{tabular}{|c|c|c|c|c|c|}
\hline & \multicolumn{2}{|c|}{ Not hypertensive } & \multicolumn{2}{|c|}{ Hypertensive } & \multirow{2}{*}{$\begin{array}{l}P, \text { for } \\
\text { trend }^{\mathrm{a}}\end{array}$} \\
\hline & Normal & Prehypertension & Stage 1 & Stage 2 & \\
\hline \multicolumn{6}{|l|}{ Men } \\
\hline Numbers & 636 & 598 & 333 & 122 & \\
\hline Smoking (\%) & 72.1 & 69.9 & 70.1 & 75.4 & 0.58 \\
\hline Drinking (\%) & 47.9 & 55.4 & 58.1 & 54.9 & 0.009 \\
\hline Diabetes (\%) & 8.5 & 15.6 & 17.9 & 23.5 & $<0.0001$ \\
\hline Age & 52.7 & 55.8 & 59.4 & 62.7 & $<0.0001$ \\
\hline $\begin{array}{l}\text { Body mass index } \\
\left(\mathrm{kg} / \mathrm{m}^{2}\right)\end{array}$ & 22 & 23.2 & 24 & 24.3 & $<0.0001$ \\
\hline $\begin{array}{l}\text { Total cholesterol } \\
(\mathrm{mg} / \mathrm{dl})\end{array}$ & 185.6 & 192.1 & 201.7 & 205.8 & $<0.0001$ \\
\hline LDL-C (mg/dl) & 124.2 & 133 & 143.5 & 145.7 & $<0.0001$ \\
\hline HDL-C (mg/dl) & 47.7 & 46.4 & 44.3 & 44.6 & 0.0003 \\
\hline Triglyceride (mg/dl) & 107.6 & 134.9 & 152.1 & 149 & $<0.0001$ \\
\hline Regular exercise (\%) & 12.7 & 16.2 & 17.5 & 20.5 & 0.10 \\
\hline Marital status (\%) & & & & & 0.005 \\
\hline Single & 4.3 & 5.1 & 4.5 & 9.8 & \\
\hline Living with spouse & 91.5 & 89.2 & 89.0 & 78.7 & \\
\hline Divorced or separate & 4.2 & 5.7 & 6.5 & 11.5 & \\
\hline Education level (\%) & & & & & 0.48 \\
\hline$<9$ years & 92.1 & 90.0 & 92.5 & 91.0 & \\
\hline$\geq 9$ years & 7.9 & 10.0 & 7.5 & 9.0 & \\
\hline Job status (\%) & & & & & $<0.0001$ \\
\hline No job & 24.7 & 29.3 & 38.7 & 47.5 & \\
\hline Blue collar & 59.6 & 51.2 & 42.8 & 41.0 & \\
\hline White collar & 15.7 & 19.6 & 18.5 & 11.5 & \\
\hline \multicolumn{6}{|l|}{ Women } \\
\hline Numbers & 697 & 574 & 429 & 186 & \\
\hline Smoking (\%) & 5.3 & 3.8 & 7 & 6.5 & 0.15 \\
\hline Drinking (\%) & 10.8 & 10.4 & 7.9 & 3.2 & 0.008 \\
\hline Diabetes (\%) & 5.6 & 13.7 & 19.8 & 20 & $<0.0001$ \\
\hline Age & 47.9 & 54.6 & 59.5 & 63.5 & $<0.0001$ \\
\hline $\begin{array}{l}\text { Body mass index } \\
\left(\mathrm{kg} / \mathrm{m}^{2}\right)\end{array}$ & 22.8 & 24 & 25.1 & 25.1 & $<0.0001$ \\
\hline $\begin{array}{l}\text { Total cholesterol } \\
\text { (mg/dl) }\end{array}$ & 192 & 206.8 & 211.4 & 212.3 & 0.06 \\
\hline LDL-C (mg/dl) & 130.6 & 145.5 & 152.1 & 152.6 & 0.004 \\
\hline $\mathrm{HDL}-\mathrm{C}(\mathrm{mg} / \mathrm{dl})$ & 50.6 & 49.1 & 46.5 & 43.5 & $<0.0001$ \\
\hline Triglyceride (mg/dl) & 100.4 & 122.7 & 152.1 & 147.8 & $<0.0001$ \\
\hline Regular exercise (\%) & 12.4 & 13.2 & 13.4 & 17.6 & 0.64 \\
\hline Marital status (\%) & & & & & $<0.0001$ \\
\hline Single & 1.3 & 1.4 & 0.0 & 1.1 & \\
\hline Living with spouse & 90.2 & 81.1 & 78.0 & 68.7 & \\
\hline Divorced or separate & 8.6 & 17.5 & 22.0 & 30.3 & \\
\hline Education level (\%) & & & & & 0.001 \\
\hline$<9$ years & 94.7 & 97.6 & 98.3 & 98.9 & \\
\hline$\geq 9$ years & 5.3 & 2.4 & 1.7 & 1.1 & \\
\hline Job status (\%) & & & & & $<0.0001$ \\
\hline No job & 55.3 & 70.4 & 78.9 & 81.7 & \\
\hline Blue collar & 24.7 & 17.0 & 12.2 & 8.6 & \\
\hline White collar & 19.9 & 12.6 & 8.9 & 9.7 & \\
\hline Menopause (\%) & 41.0 & 65.9 & 76.2 & 87.0 & $<0.0001$ \\
\hline
\end{tabular}

LDL-C, low-density lipoprotein cholesterol; HDL-C, high-density lipoprotein cholesterol. ${ }^{a}$ Test for linear trend across blood pressure categories after age adjustment. For dichotomous variables, logistic regression was applied. 
significantly as blood pressure categories shifted from normotensive to prehypertension to hypertension. HDL-C levels decreased significantly and progressively. The smoking prevalence rates did not change among different categories, and drinking rates decreased. Diabetes rates increased as blood pressure increased. Women had similar patterns of risk factor prevalence as men, with a lower prevalence of smoking and drinking rates. Hypertensive participants had a lower rates of living with a spouse and being of white collar status, in both genders. The proportion of women at menopause was higher in the population of women with hypertension.

The odds ratios and $95 \%$ confidence intervals of various covariates for developing hypertension are presented in Table 2. Different periods had a significant effect on the incidence rates; the odds ratios increased from 2.30 to 20.1 in the second and fourth periods versus the first period. Women had an $18 \%$ lower risk of developing hypertension than men. Each year increase in age or each increase in one unit of BMI was associated with a $3 \%$ increase in risk. Baseline prehypertension increased the risk by $71 \%$ compared to normotensive status, and metabolic syndrome increased the risk of hypertension by $35 \%$. Smoking elicited $19 \%$ less risk for hypertension incidence, and alcohol drinking increased the risk of hypertension incidence by $14 \%$. The association of baseline prehypertension and further hypertension was not modified as a result of BMI level, or the presence or absence of other covariate status. Figure 1 shows the adjusted cumulative probability of hypertension incidence among different visit periods, specified by baseline normotensive or prehypertensive status. The patterns of adjusted cumulative incidence rates are curvilinear, increasing progressively in baseline normotensive subjects, from $1 \%$ in the first period to $27 \%$ in the fourth period. The pattern for prehypertensive subjects was slightly steeper, from $4 \%$ in the first period to $59 \%$ in the fourth period. The results implied that the different periods had significant effects on hypertension incidence, after adjusting for multiple covariates.

We followed those who were cardiovascular disease-free in the baseline cohort up to 2005; 332 cases developed
Fig. 1

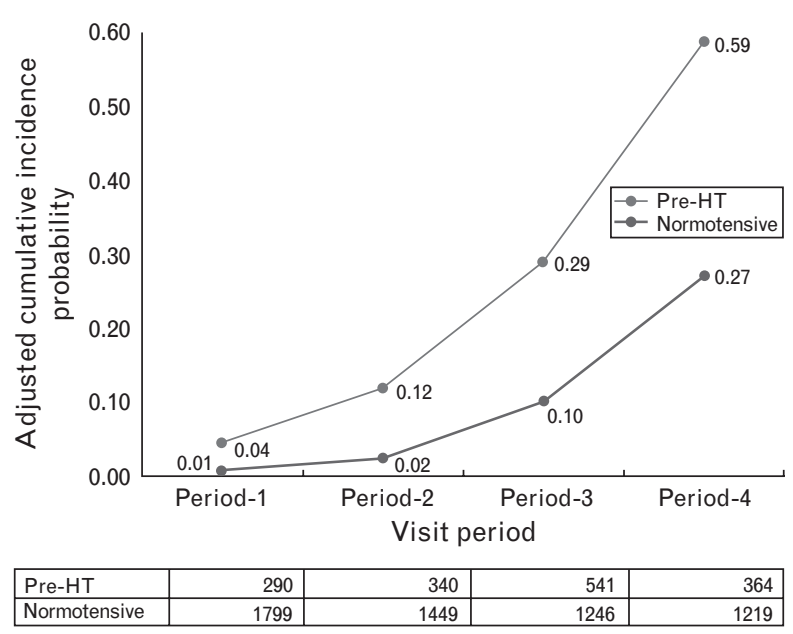

The adjusted cumulative probability proportions of hypertension incidence among different visit periods in the Chin-Shan Community Cardiovascular Cohort (CCCC) study, Taiwan, specified by baseline blood pressure. Pre-HT, prehypertensive. The adjusted variables included gender, age, body mass index, smoking, drinking and metabolic syndrome status. The numbers in the table are the number of persons in the two groups at each time period.

cardiovascular events, including 122 coronary heart disease events and 210 stroke events. The blood pressurespecific prevalences and incidence of cardiovascular events are plotted in Fig. 2. The frequency distribution patterns of systolic and diastolic blood pressure are curves skewed to the right. The incidence rates of cardiovascular events increased significantly with both systolic and diastolic blood pressure, with a slight U-shape in the extremely low diastolic blood pressure group. Kaplan-Meir curves of survival function free from cardiovascular events, specified by blood pressure categories, are plotted in Fig. 3. The survival curves were distinctly different from each other, with the log-rank test as statistically significant $(P<0.001)$. The predicted model of various risk factor profiles in predicting cardiovascular events is presented in Table 3. Age and male gender were significant risk factors, and diabetes mellitus elicited $97 \%$ risk, left ventricular hypertrophy $36 \%$ risk and hypercholesterolemia $47 \%$ risk for cardiovascular events. Baseline blood pressure categories

Table 2 Odds ratios for development of hypertension in the Chin-Shan Community Cardiovascular Cohort (CCCC) study, Taiwan

\begin{tabular}{|c|c|c|c|c|c|}
\hline Covariate & & Odds ratio & Low 95\% Cl & High $95 \% \mathrm{Cl}$ & $P$ value \\
\hline \multirow{3}{*}{ Period effect } & 4 versus 1 & 20.12 & 15.05 & 26.91 & $<0.0001$ \\
\hline & 3 versus 1 & 7.42 & 6.22 & 8.86 & $<0.0001$ \\
\hline & 2 versus 1 & 2.30 & 1.94 & 2.73 & $<0.0001$ \\
\hline Gender & Women versus men & 0.82 & 0.68 & 0.99 & 0.037 \\
\hline Age & +1 year & 1.03 & 1.02 & 1.03 & $<0.0001$ \\
\hline Body mass index & $+1 \mathrm{~kg} / \mathrm{m}^{2}$ & 1.03 & 1.01 & 1.06 & 0.011 \\
\hline Baseline blood pressure status & Pre-HT versus. normotensive & 1.71 & 1.47 & 1.98 & $<0.0001$ \\
\hline Smoking status & Yes versus no & 0.81 & 0.67 & 0.98 & 0.029 \\
\hline Drinking status & Yes versus no & 1.14 & 0.98 & 1.34 & 0.098 \\
\hline Metabolic syndrome & Yes versus no & 1.35 & 1.11 & 1.65 & 0.003 \\
\hline
\end{tabular}

$\mathrm{Cl}$, confidence interval; Pre-HT, prehypertensive. 
Fig. 2

(a)

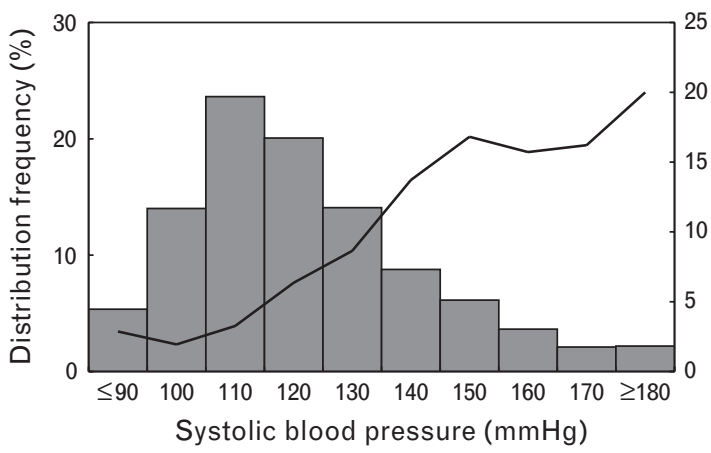

(b)

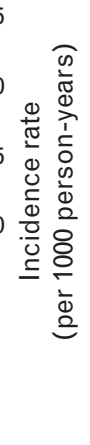

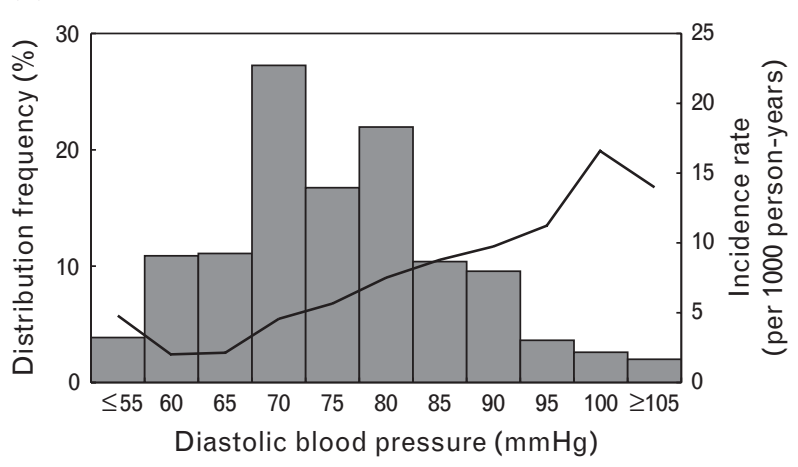

Blood pressure-specific prevalences (histogram) and cardiovascular disease incidence rates (line) in the Chin-Shan Community Cardiovascular Cohort (CCCC) study, Taiwan. (a) Systolic blood pressure, (b) diastolic blood pressure.

Fig. 3

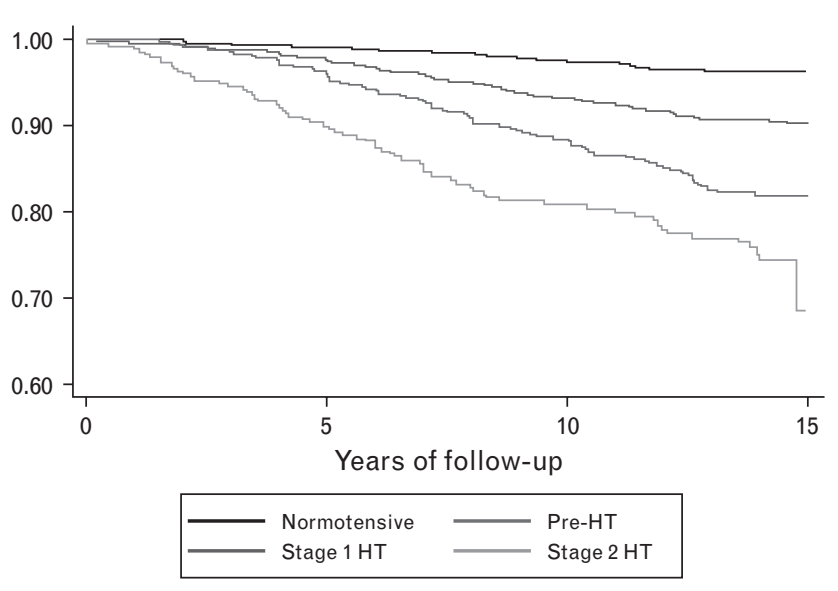

Kaplan-Meir survival curves for cardiovascular events in the study population, specified by baseline blood pressure categories. Pre-HT, prehypertension; HT, hypertension. played important roles in predicting cardiovascular risk, and the hazard risks of prehypertension and hypertension increased from 1.73 to 4.52 , compared with baseline normotensive subjects.

\section{Discussion}

New onset of hypertension is a frequent phenomenon among the middle-aged population. The incidence rates of hypertension were strongly related with general obesity and baseline blood pressure categories in this Taiwanese cohort. Hypertension also, played an important role in predicting cardiovascular events. The study results show clearly high incidence rates of hypertension among ethnic Chinese.

Previous studies have provided important risk factors for hypertension incidence events, such as socio-economic status [8], white blood cell count [3], uric acid [7] and fasting insulin levels [5]. Among these risk factors, obesity and metabolic syndrome are crucial in predicting further hypertension incidence. Our study clearly demonstrated that obesity and metabolic syndrome

Table 3 Model for risk factor profiles to predict cardiovascular events in the Chin-Shan Community Cardiovascular Cohort (CCCC) study, Taiwan

\begin{tabular}{|c|c|c|c|c|}
\hline Risk factors & Hazard risk & Lower $95 \% \mathrm{Cl}$ & Upper $95 \% \mathrm{Cl}$ & $P$ value \\
\hline Age $(+1$ year $)$ & 1.06 & 1.04 & 1.07 & $<0.0001$ \\
\hline Gender (women versus men) & 0.46 & 0.30 & 0.71 & 0.000 \\
\hline Body mass index $\left(+1 \mathrm{~kg} / \mathrm{m}^{2}\right)$ & 1.04 & 0.99 & 1.09 & 0.114 \\
\hline Normal blood pressure & 1 (reference) & & & \\
\hline Prehypertension & 1.73 & 1.00 & 2.98 & 0.050 \\
\hline Stage 1 hypertension & 4.17 & 2.45 & 7.11 & $<0.0001$ \\
\hline Stage 2 hypertension & 4.52 & 2.45 & 8.36 & $<0.0001$ \\
\hline Diabetes mellitus (yes versus no) & 1.97 & 1.36 & 2.83 & 0.000 \\
\hline Cholesterol $\geq 240 \mathrm{mg} / \mathrm{dl}$ & 1.47 & 1.05 & 2.06 & 0.024 \\
\hline LV hypertrophy (yes versus no) & 1.36 & 0.89 & 2.07 & 0.156 \\
\hline Smoking (yes versus no) & 0.91 & 0.60 & 1.38 & 0.667 \\
\hline Drinking (yes versus no) & 0.93 & 0.64 & 1.37 & 0.727 \\
\hline
\end{tabular}

$\mathrm{Cl}$, confidence interval; LV, left ventricular. 
significantly impact risk for hypertension. Obesity indices, such as general or abdominal obesity, were related with prehypertension and insulin resistance [23]. Optimal body weight control and reduction of metabolic syndrome risk may have beneficial effects on hypertension control. Health promotion programs, such as weight reduction and physical activity, will help to prevent hypertension.

Baseline hypertension elicited significant burdens on cardiovascular events in this prospective study, and the evidence showed that traditional risk factors, such as hypertension, obesity, hyperlipidemia and diabetes mellitus, have great effects on cardiovascular events. Left ventricular hypertrophy added additional risk to the prognosis of cardiovascular events. In addition, prehypertension was clustered with atherosclerotic risk factors, such as obesity, insulin resistance and various cardiometabolic risk factors [13]. The great impact of prehypertension on hypertension incidence, compared with normotensive subjects, implied that optimal control of prehypertension is mandatory for prevention of cardiovascular disease events. Our study reconfirmed the previous studies about prehypertension risk on cardiovascular events in Western countries [24-26]. In the Framingham cohort data, the 4-year progression rates of hypertension were $37.3 \%$ in the high-normal category in subjects aged less than 65 years and $49.5 \%$ in those older than 65 years [24]. The Framingham Heart study also showed that the lifetime cumulative incidence for hypertension reached 90\% among middle-aged participants [25]. These substantial cumulative values were due to the combined effects of age, gender, obesity and lifestyle risk factors. Our study also estimated that the crude cumulative probability reached $70 \%$ among normotensive and $83 \%$ among prehypertensive subjects, after 6 years' follow-up. Furthermore, after adjusting these risk factors, the period effects still elicited significant risks on incidence of hypertension, although the cumulative incidence probabilities were attenuated (Fig. 1). Recent randomized controlled trial data demonstrated that pharmacotherapy of prehypertension blocked the progress into hypertension and potentially reduced the cardiovascular event risks [27].

We clearly demonstrated that several atherosclerotic risk factors could predict the further cardiovascular events in the ethnic Chinese population, such as age, gender, blood pressure, diabetes mellitus and hypercholesterolemia. The results implied the importance of comprehensive programs to prevent cardiovascular events.

Hypertensive women had non-significantly higher antihypertensive medication than hypertensive men (32.4 versus $27.5 \%, P=0.103$ ), implying that hypertension control should be monitored closely within the community population.
This study has several strengths. First, we clearly demonstrated the impact of various blood pressure categories on cardiovascular events in the ethnic Chinese community. Second, we specified prehypertension status as a separate group and we clearly demonstrated that prehypertension status elicited significant risk of further cardiovascular events. The fact of gradual dose-response blood pressure risk should be emphasized in the community. Community-based prevention programs for hypertension control should be implemented for prevention of cardiovascular risk [28].

There are several limitations to this study. First, the study sample was limited to the middle-aged and older populations in the community, and the high prevalence and incidence rates of hypertension might have limited the extrapolation of the results to the general ethnic Chinese population. Our study showed that more than $70 \%$ of study subjects developed hypertension during the follow-up decade. Our sample might be too small to elucidate the role of prehypertension alone or its transition to more severe hypertension for cardiovascular morbidity. Second, we did not incorporate several important confounding factors, such as physical activity and inflammation biomarkers into the model, in order to estimate the effects of confounding factors. Also, we did not collect the details of antihypertension medication history in order to explore the effects of various medications. These limitations can be resolved by a randomized, controlled trial design, and one recent report provided hints about the beneficial effects of prehypertension management [27].

In conclusion, the hypertension incidence rates among middle-aged and older ethnic Chinese are high, associated with obesity and metabolic syndrome status. Furthermore, the baseline prehypertension elicits a significant risk for cardiovascular events in the community. Optimal blood pressure control and risk factor reduction by lifestyle intervention will be crucial for reducing new-onset hypertension and further cardiovascular events among the ethnic Chinese population.

\section{Acknowledgements}

This study was supported partly by grants from the National Science Council (NSC 94-2314-B-002-290, NSC 93-2314-B-002-218) in Taiwan. The authors wish to thank the cardiologists at National Taiwan University Hospital for their invaluable assistance.

There are no conflicts of interest.

\section{References}

1 Kannel WB. Risk factors in hypertension. J Cardiovasc Pharmacol 1989; 13 (Suppl 1):S4-S10.

2 Cornoni-Huntley J, LaCroix AZ, Havlik RJ. Race and sex differentials in the impact of hypertension in the United States. The National Health and Nutrition Examination Survey I Epidemiologic Follow-up Study. Arch Intern Med 1989; 149:780-788. 
3 Gillum RF, Mussolino ME. White blood cell count and hypertension incidence. The NHANES I Epidemiologic Follow-up Study. J Clin Epidemiol 1994; 47:911-919.

4 He J, Klag MJ, Appel LJ, Charleston J, Whelton PK. Seven-year incidence of hypertension in a cohort of middle-aged African Americans and whites. Hypertension 1998; 31:1130-1135.

5 He J, Klag MJ, Caballero B, Appel LJ, Charleston J, Whelton PK. Plasma insulin levels and incidence of hypertension in African Americans and whites. Arch Intern Med 1999; 159:498-503.

6 Pereira MA, Folsom AR, McGovern PG, Carpenter M, Arnett DK, Liao D, et al. Physical activity and incident hypertension in black and white adults: the Atherosclerosis Risk in Communities Study. Prev Med 1999; 28:304312.

7 Sundstrom J, Sullivan L, D'Agostino RB, Levy D, Kannel WB, Vasan RS. Relations of serum uric acid to longitudinal blood pressure tracking and hypertension incidence. Hypertension 2005; 45:28-33.

8 Vargas CM, Ingram DD, Gillum RF. Incidence of hypertension and educational attainment: the NHANES I epidemiologic followup study. First National Health and Nutrition Examination Survey. Am J Epidemiol 2000; 152:272-278.

9 Chobanian AV, Bakris GL, Black HR, Cushman WC, Green LA, Izzo JL Jr, et al. The Seventh Report of the Joint National Committee on Prevention, Detection, Evaluation, and Treatment of High Blood Pressure: the JNC 7 report. JAMA 2003; 289:2560-2572.

10 Chien KL, Hsu HC, Sung FC, Su TC, Chen MF, Lee YT. Hyperuricemia as a risk factor on cardiovascular events in Taiwan: The Chin-Shan Community Cardiovascular Cohort Study. Atherosclerosis 2005; 183:147-155.

11 Chien KL, Sung FC, Hsu HC, Su TC, Chang WD, Lee YT. Relative importance of atherosclerotic risk factors for coronary heart disease in Taiwan. Eur J Cardiovasc Prev Rehabil 2005; 12:95-101.

12 Lee YT, Lin RS, Sung FC, Yang CY, Chien KL, Chen WJ, et al. Chin-Shan Community Cardiovascular Cohort in Taiwan: baseline data and five-year follow-up morbidity and mortality. J Clin Epidemiol 2000; 53:836-846.

13 Govindarajan G, Whaley-Connell A, Mugo M, Stump C, Sowers JR. The cardiometabolic syndrome as a cardiovascular risk factor. Am J Med Sci 2005; 330:311-318.

14 Chien KL, Sung FC, Hsu HC, Su TC, Lin RS, Lee YT. Apolipoprotein A1 and $\mathrm{B}$, and stroke events in a community-based cohort in Taiwan: Report of Chin-Shan Community Cardiovascular Study. Stroke 2002; 33:39-44.

15 Adult Treatment Panel III. Third Report of the National Cholesterol Education Program (NCEP) Expert Panel on Detection, Evaluation, and Treatment of High Blood Cholesterol in Adults (Adult Treatment Panel III) final report. Circulation 2002; 106:3143-3421.

16 Tan CE, Ma S, Wai D, Chew SK, Tai ES. Can we apply the National Cholesterol Education Program Adult Treatment Panel definition of the metabolic syndrome to Asians? Diabetes Care 2004; 27:1182-1186.

17 Hosmer DW Jr, Lemeshow S. Extensions of the proportional hazards model. Applied survival analysis: Regression modeling of time to event data. New York: John Wiley \& Sons; 1999; pp. 241-270.

18 Collett D. Interval-censored survival data. Modelling survival data in medical research, 2nd ed. Boca Raton: Chapman \& Hall/CRC; 2003; pp. 273-298.

19 Kaplan EL, Meier P. Nonparametric estimation from incomplete observations. J Am Stat Assoc 1958; 53:457-481.

20 Cox DR. Regression models and life-tables. J R Stat Soc B 1972; 34:187202.

21 Lin DY, Wei $\sqcup$. The robust inference for the Cox proportional hazards model. J Am Stat Assoc 1989; 84:1074-1078.

22 Grambsch PM, Therneau TM. Proportinal hazards test and diagnostics based on weighted residuals. Biometrika 1994; 81:515-526.

23 Okosun IS, Boltri JM, Anochie LK, Chandra KM. Racial/ethnic differences in prehypertension in American adults: population and relative attributable risks of abdominal obesity. J Hum Hypertens 2004; 18:849-855.

24 Vasan RS, Larson MG, Leip EP, Kannel WB, Levy D. Assessment of frequency of progression to hypertension in non-hypertensive participants in the Framingham Heart Study: a cohort study. Lancet 2001; 358:16821686.

25 Vasan RS, Beiser A, Seshadri S, Larson MG, Kannel WB, D'Agostino RB, Levy D. Residual lifetime risk for developing hypertension in middle-aged women and men: The Framingham Heart Study. JAMA 2002; 287:10031010.

26 Vasan RS, Larson MG, Leip EP, Evans JC, O'Donnell CJ, Kannel WB, Levy $\mathrm{D}$. Impact of high-normal blood pressure on the risk of cardiovascular disease. N Engl J Med 2001; 345:1291-1297.

27 Julius S, Nesbitt SD, Egan BM, Weber MA, Michelson EL, Kaciroti N, et al. Feasibility of treating prehypertension with an angiotensin-receptor blocker. N Engl J Med 2006; 354:1685-1697.
28 Whelton PK, He J, Appel LJ, Cutler JA, Havas S, Kotchen TA, et al. Primary prevention of hypertension: clinical and public health advisory from The National High Blood Pressure Education Program. JAMA 2002; 288:1882-1888. 